\title{
EFFICACY OF INTERFERON-INDUCIBLE PROTEIN-10 FOR MONITORING STANDARD TUBERCULOSIS TREATMENT
}

\author{
*Alaa Refaat, *Nasr Affara, * Abeer Alhawari, and **Mohamed Fathi \\ *Chest and **Clinical Pathology Departments, Faculty of Medicine, Zagazig University, Egypt.
}

\begin{abstract}
Introduction: Tuberculosis (TB) remains a major global health problem. Interferon-inducible Protein-10 (IP-10) has been proposed as a promising biomarker for monitoring the efficacy of standard therapy in patients with active pulmonary TB. Objective: The propose of this study was to assess the efficacy of IP-10 in patients with active pulmonary TB as a sign of a response to TB standard therapy. Patients and methods: A total of 136 newly diagnosed pulmonary tuberculosis patients were recruited in this study. The patients were classified into 2 groups according to the presence of the risk factors of relapse. Group (1) included 30 patients with low risk of relapse and group (2) included 25 patients with moderate and high risk of relapse. In this study, we assessed the IP-10 levels in both serum and in response to QuantiFeron-TB Gold In-Tube test (QFT-GIT) antigens at time of diagnosis, at month 2 and after completing treatment. Results: IP-10 median levels in both serum and in response to QFT-GIT antigens were not significantly different between both patient groups at baseline, at month 2 and after completion of TB treatment. However, in each patient group, medians of IP-10 levels before treatment decreased significantly with time at 2 months and after the completion of anti-TB treatment (all P < 0.05). Conclusion: Interferon-inducible Protein-10 (IP-10) in serum or in response to QFT-GIT antigens was a useful biomarker for monitoring the efficacy of the standard regimen in patients with active pulmonary TB.
\end{abstract}

Keywards: Interferon-inducible Protein-10, Monitoring, Tuberculosis.

Abbreviations: Interferon-inducible Protein-10 (IP-10), QuantiFeron-TB Gold In-Tube test (QFT-GIT), Tuberculosis (TB), Acid-fast bacilli (AFB), Tuberculin test (TST), Chest X-ray (CXR).

\section{INTRODUCTION}

$\mathbf{T}$ uberculosis (TB) remains a major health problem worldwide [1]. Relapse of TB still considered as a significant burden on TB control programs. Therefore, predication of patients with increased risk of poor outcome may potentially reduce this burden [2].

Patients with radiological cavitary lesion and positive smear/culture for Mycobacterium tuberculosis after two months of antituberculous are more risky for relapse to more than $20 \%$ within the following two years, compared with $5 \%$ for patients with one risk factor and only $2 \%$ for patients without risk factors [3]. Extension of the continuation phase from four to seven months can decrease the relapse rate in high risk cases[4]. In the course of monitoring of TB treatment, sputum conversion after 2 months is not always predictive of a cure [5]. Therefore, surrogate biomarkers that can predict the treatment response are urgently needed. These biomarkers would be of value in monitoring of these patients by serving a clinical judgment on the need of prolonged regimens, and in enhancement of drug development process by serving a validated surrogate endpoints.

Recently, Interferon gamma inducible protein-10 (IP-10) is emerged as a promising biomarker for diagnosis of TB. IP-10 is a proinflamatory chemokine secreted by inflammatory cells in response to IFN- $\gamma$, which is secreted by antigen specific T-lymphocytes. IP-10 is involved in trafficking monocytes and activated T-helper cell type 1 cells to inflamed foci [6]. There have been conflicting results about IP-10 as a diagnostic tool anf if can differentiate between active and latent tuberculosis . Higher levels of IP10 were recently observed in antigen stimulated TB blood samples, which decrease significantly upon TB treatment [7]. However, the clinical value of TB antigen-stimulated IP-10 and serum IP-10 for predicting treatment outcome not extensively investigated. The objective of this study was to assess the efficacy of IP-10 in patients with active pulmonary $\mathrm{TB}$ as a sign of response to TB standard therapy.

\section{PATIENTS AND METHODS}

This study was done at Respirology Department and Clinical Pathology Department, Farwaniya Hospital, Tuberculosis Centre, and Alrashed Rrespiratory Centre, Ministry of Health, State of Kuwait, in the period between June 2013 and October 2014. A total of 136 newly diagnosed pulmonary tuberculosis patients were recruited. The patients were classified according to the presence of the following risk factors of relapse: 1) the presence of a cavitary lesion on chest X-ray or chest computed tomography (CT) and/or 2) a 
positive sputum culture after 2 months of anti-TB treatment [8]. Group (1) included 30 patients with low risk without any risk factor of relapse and group (2) included 25 patients with moderate and high risk of relapse. High risk was defined if both risk factors were existing, while the moderate risk of the presence of one risk factor alone.

\section{Sputum collection and processing}

Early-morning sputum specimens were collected at the start and the end of anti-TB treatment for sputum smear and culture conversion and also collected at the end of the intensive phase (month 2) and at the third month (if the specimen obtained at month 2 is smearpositive). All collected samples were digested using N-acetyl-L-cysteine-sodium hydroxide (NALC-NaOH) solution. Then the mixture was neutralized with phosphate buffered saline and centrifuged at 3,000 rpm for 15 minutes.. The supernatant was poured off, the resulting sediment was re-suspended in $1 \mathrm{ml}$ phosphate buffered saline and the suspension was used for smear preparation and inoculation in culture [9]. All sputum samples that collected from each patient were positive for acid fast bacilli (AFB) by ZiehlNeelsen method and culture in Lowenstein-Jensen media. The presence of Mycobacterium tuberculosis in the positive culture samples were further confirmed by real-time PCR method (Primedesign Ltd, Southampton, UK). Sputum grading was done based on the number of AFB in the smear, according to American Thoracic Society and Centers for Disease Control and Prevention recommendations [10] (table,1).

\section{Radiological evaluations:}

Standard posterio-anterior chest radiography was done at time of diagnosis and after two and six months of antituberculous treatment for all enrolled patients. The CT was performed at the time of diagnosis to assess the extent of the lesions and the presence of typical TB features such as cavitation, centrilobular nodules, and consolidation. The proportion of the affected lung was performed by a visual estimate of the extent of parenchyma infiltrates; a proportion of $30 \%$ of affected lung was used as cutoff value to grade TB severity. Each lung was divided into two zones (upper and lower zones). Upper zones were defined as the area of the lung above the level of carina. The disease was graded using a sliding scale of severity as follows: 0 : normal chest $\mathrm{X}$-rays; 1 : mild grade (fibrosis and/or nodules only); 2: low grade (infiltrates with proportion of lung affected $<30 \%$ ); 3: intermediate grade (infiltrates with proportion of lung affected $>30 \%$ and/or cavitation $<4 \mathrm{~cm}$ in diameter); 4 : High grade (an infiltrate of any percentage of extension with cavitation $>4 \mathrm{~cm}$ in diameter and/or bronchial spread and/or miliary and/or pleural effusion, and/or adenopathy) [11].

\section{Tuberculin skin test:}

The 5 TU (tuberculin unit) of purified protein derivative (PPD) RT23 (Statens Serum Institute, Copenhagen, Denmark) was injected intradermally using Mantoux method. The reaction was evaluated by measuring the diameter of induration between 48 and 72 hours. The cutoff point for tuberculin test positivity was considered as $10 \mathrm{~mm}$.

\section{QFT-GIT assay:}

QuantiFeron-TB Gold In-Tube test (QFTGIT) was performed by collecting $1 \mathrm{ml}$ of whole heparinized blood in 3 tubes, one containing only heparin as a negative control (nil tube), and Phytohaemagglutinin (PHA) as a positive mitogen control. The third tube containing 3 Mycobacterium tuberculosis specific antigens: ESAT-6, CFP-10 and TB 7.7. Tubes were incubated at $37^{\circ} \mathrm{C}$ for 24 hours and centrifuged. The supernatant plasma was collected for testing. The QFT-GIT assay was considered positive if IFN- $\gamma$ was $0.35 \mathrm{IU} / \mathrm{ml}$ in the antigenstimulated tube after subtracting the level in the nil tube. The test was done according to the manufacturer's protocol (Cellestis, Carnegie, Australia).

\section{Measurement of IP-10:}

The IP-10 level was measured in a duplicate in supernatants collected from the plasma of QFTGIT tubes and in sera from all patients, using human CXCL10/IP-10 Quantikine ELISA Kit in accordance with the manufacturer's instructions (R\&D Systems, Minneapolis, MN, USA). Blood sampling for both IP-10 measurement and QFTGIT assay was performed at the time of diagnosis ( 0 month), after 2 months of antiTB treatment ( 2 months), and after the completion of anti-TB treatment (Post-Tx).

\section{TB treatment:}

All enrolled patients received standard antituberculous treatment based on guidelines for Tuberculosis 2011[12]. The standard regimen included 2-month intensive phase of 
rifampicin (10 $\mathrm{mg} / \mathrm{kg} /$ day $)$, isoniazid (5 $\mathrm{mg} / \mathrm{kg} /$ day), ethambutol (15-25 $\mathrm{mg} / \mathrm{kg} /$ day $)$, and pyrazinamide (15-30 $\mathrm{mg} / \mathrm{kg} /$ day $)$, subsequently, the 4-month continuation phase included isoniazid and rifampin administration. Every patient received regular follow-up at our chest outpatient clinics. The duration of treatment was at least 6 months, depended on the relapse risk, the clinical and radiological response to treatment, and the presence of drug intolerance.

Exclusion criteria

All patients with resistance to first-line antiTB drugs, previous history of TB, or those with documented immunosurpressive disease such as HIV infection, end-stage renal disease, leukemia/lymphoma or who were undergoing immunosuppressive therapy were excluded from the study.

\section{Statistical analysis}

Statistical analysis was done using statistical software 'SPSS version 10.0 (SPSS Inc, Chicago, IL, USA). Normally distributed variables were summarized as mean with standard deviation and compared between the two groups using Student's t-test. Chi-squared test and Fisher exact test were applied to compare the ordinal variables. For all analyses, $p$ value $<0.05$ was considered statistically significant and $\mathrm{p}$ value < 0.001 was considered highly statistically significant.

Table 1: Sputum-smear grading:

\begin{tabular}{lccc}
\hline If the slide has: & $\begin{array}{c}\text { No. of fields to } \\
\text { be examined }\end{array}$ & Grading & Results \\
\hline No AFB in 100 fields & 100 & 0 & Negative \\
\hline 1-9 AFB per 100 fields & 100 & Scanty & Positive \\
\hline 10-99 AFB per 100 fields & 100 & $1+$ & Positive \\
\hline 1-10 AFB per field & 50 & $2+$ & Positive \\
\hline More than 10 AFB per field & 20 & $3+$ & Positive \\
\hline
\end{tabular}


Table (2): Baseline clinical and laboratory characteristics of both patient groups:

\begin{tabular}{|c|c|c|c|c|}
\hline & & $\begin{array}{c}\text { Group (1) } \\
\text { (Low-risk) }\end{array}$ & $\begin{array}{c}\text { Group (2) } \\
\text { (Moderate/High- } \\
\text { risk) }\end{array}$ & $\begin{array}{c}\mathbf{P}- \\
\text { Value }\end{array}$ \\
\hline No. & & $30 / 55(54.5 \%)$ & $25 / 55(45.5 \%)$ & - \\
\hline Age, years $($ mean $\pm S D)$ & & $39 \pm 14$ & $37 \pm 10$ & $>0.05$ \\
\hline Gender & & $\begin{array}{l}\mathrm{F}=18(60 \%) \\
\mathrm{M}=12(40 \%)\end{array}$ & $\begin{array}{l}\mathrm{F}=16(64 \%) \\
\mathrm{M}=9(36 \%)\end{array}$ & $>0.05$ \\
\hline Smoking & & $8(26.7 \%)$ & $7(28 \%)$ & $>0.05$ \\
\hline COPD* & & $6(20 \%)$ & $6(2 \%)$ & $>0.05$ \\
\hline Diabetes Mellitus & & $3(10 \%)$ & $5(00 \%)$ & $>0.05$ \\
\hline TST positivity & & $23(68 \%)$ & $18(72 \%)$ & $>0.05$ \\
\hline QFT-GIT positivity & & $27(90 \%)$ & $22(88 \%)$ & $>0.05$ \\
\hline \multirow{4}{*}{$\begin{array}{l}\text { Smear AFB positivity Grade } \\
(\%)\end{array}$} & Scanty & $2(6.7 \%)$ & $2(8 \%)$ & $>0.05$ \\
\hline & $1+$ & $9(30 \%)$ & $7(28 \%)$ & $>0.05$ \\
\hline & $2+$ & $10(33.3 \%)$ & $6(24 \%)$ & $>0.05$ \\
\hline & $3+$ & $9(30 \%)$ & $10(40 \%)$ & $>0.05$ \\
\hline \multirow{5}{*}{ 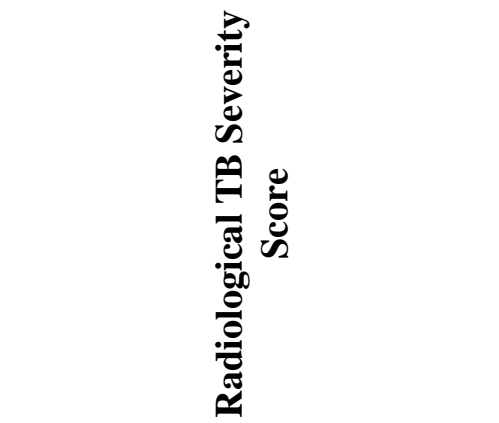 } & None & $0(0 \%)$ & $0(0 \%)$ & $>0.05$ \\
\hline & Mild & $3(10 \%)$ & $1(4 \%)$ & $>0.05$ \\
\hline & Low & $10(33.3 \%)$ & $7(28 \%)$ & $>0.05$ \\
\hline & Intermediate & $8(26.7 \%)$ & $7(28 \%)$ & $>0.05$ \\
\hline & High & $9(30 \%)$ & $10(40 \%)$ & $>0.05$ \\
\hline
\end{tabular}

- All values expressed as no (\%) unless specified.

* COPD; Chronic obstructive pulmonary disease, DM; Diabetes Mellitus. 
Table (3): Comparisons of treatment response and duration of medications between both patient groups:

\begin{tabular}{|c|c|c|c|c|}
\hline & & $\begin{array}{c}\text { Group (1) } \\
\text { (Low-risk) }\end{array}$ & $\begin{array}{l}\text { Group (2) } \\
\text { (Moderate/ }\end{array}$ & $\mathbf{P}$ - Value \\
\hline \multicolumn{2}{|l|}{ No. } & $30 / 55(54.5 \%)$ & $25 / 55(45.5 \%)$ & - \\
\hline \multicolumn{2}{|c|}{$\begin{array}{l}\text { Sputum conversion after } 2 \text { months (culture- } \\
\text { based) }\end{array}$} & $29(96.7 \%)$ & $20(80 \%)$ & $<0.05$ \\
\hline \multicolumn{2}{|c|}{ Delayed resolution on CXR at month 2} & $6(20 \%)$ & $11(44 \%)$ & $<0.05$ \\
\hline \multicolumn{2}{|c|}{ Extended treatment $>6$ months } & $3(10 \%)$ & $7(28 \%)$ & $<0.05$ \\
\hline \multirow{4}{*}{$\begin{array}{l}\text { Duration of medications } \\
\text { (months) }(\text { mean } \pm \text { SD) }\end{array}$} & Isoniazid & $7.3 \pm 1.7$ & $8.9 \pm 2.3$ & $>0.05$ \\
\hline & Rifampin & $7.3 \pm 1.7$ & $8.2 \pm 3.1$ & $>0.05$ \\
\hline & Ethambutol & $3.6 \pm 6$ & $4.8 \pm 1$ & $>0.05$ \\
\hline & Pyrazinamide & $2.2 \pm 1$ & $2.7 \pm 3$ & $>0.05$ \\
\hline
\end{tabular}

- All values expressed as no (\%) unless specified

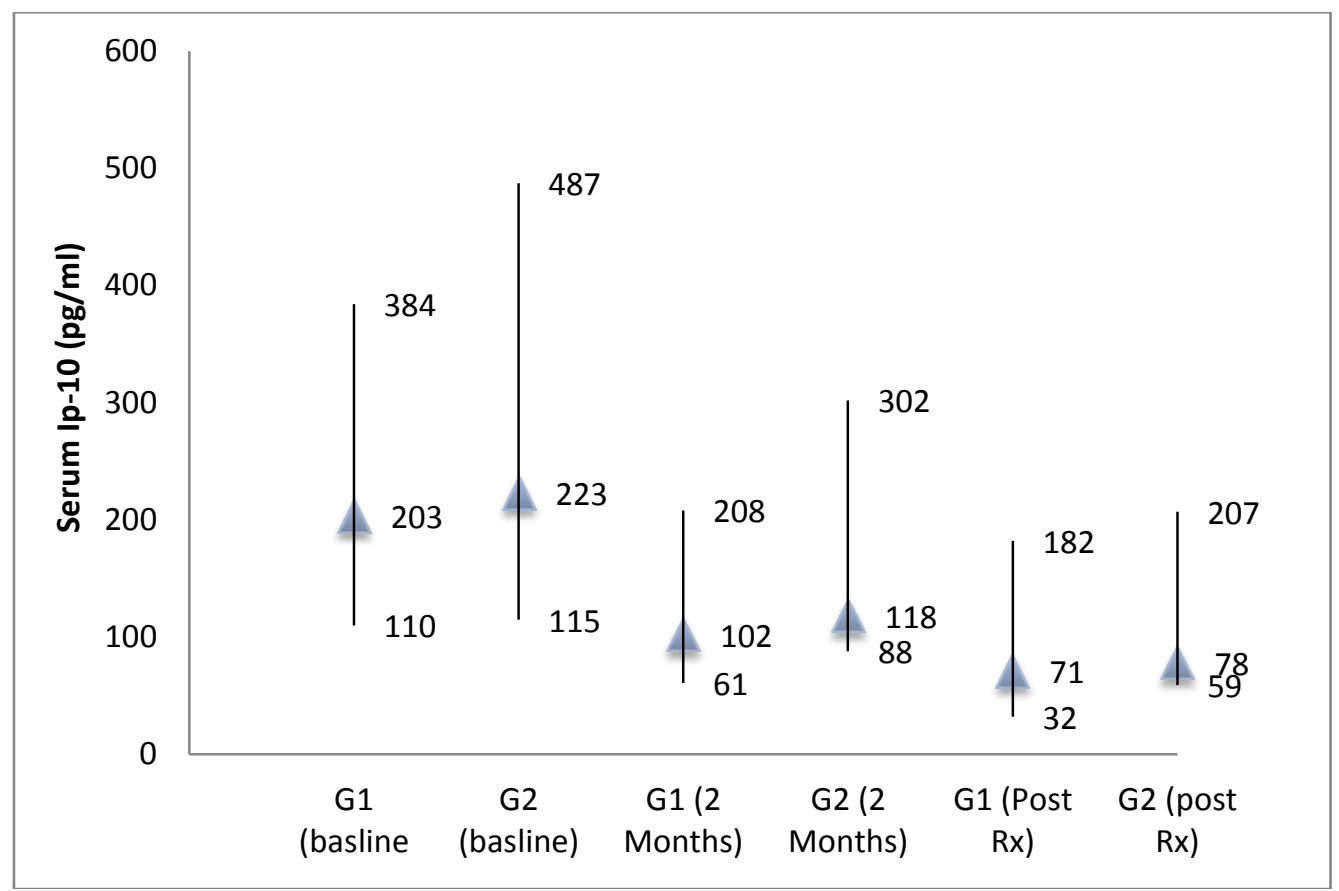

Figure (1): Boxplots showing medians and interquartile ranges of serum IP-10 in both patients groups at baseline sampling, at month 2 and at completion of therapy, ( $G=G r o u p)$. 

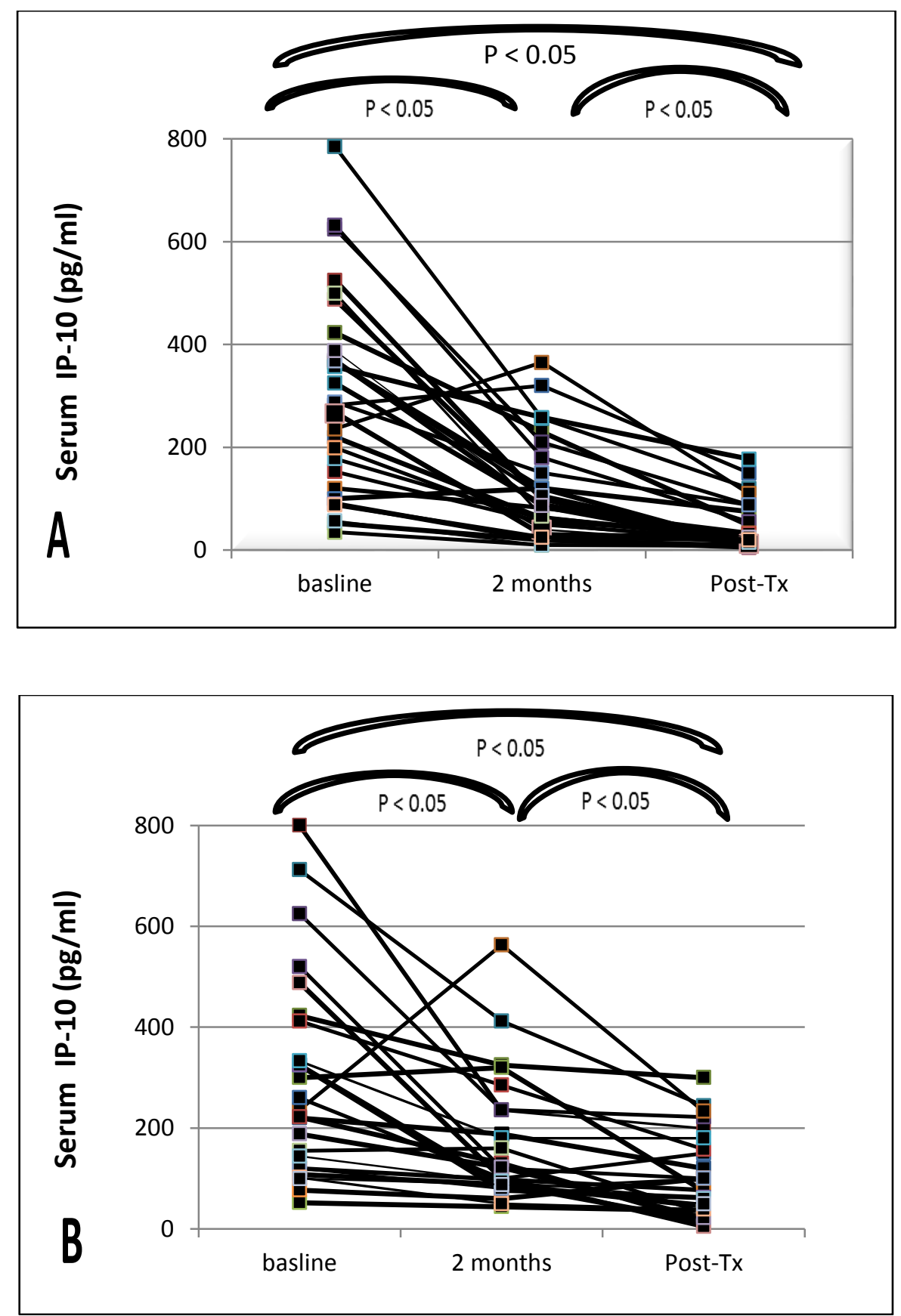

Figure (2): Longitudinal analysis of Serum IP-10 levels in patients of both groups (group 1; A, group 2; B) at baseline sampling, at month 2 and at completion of therapy. 


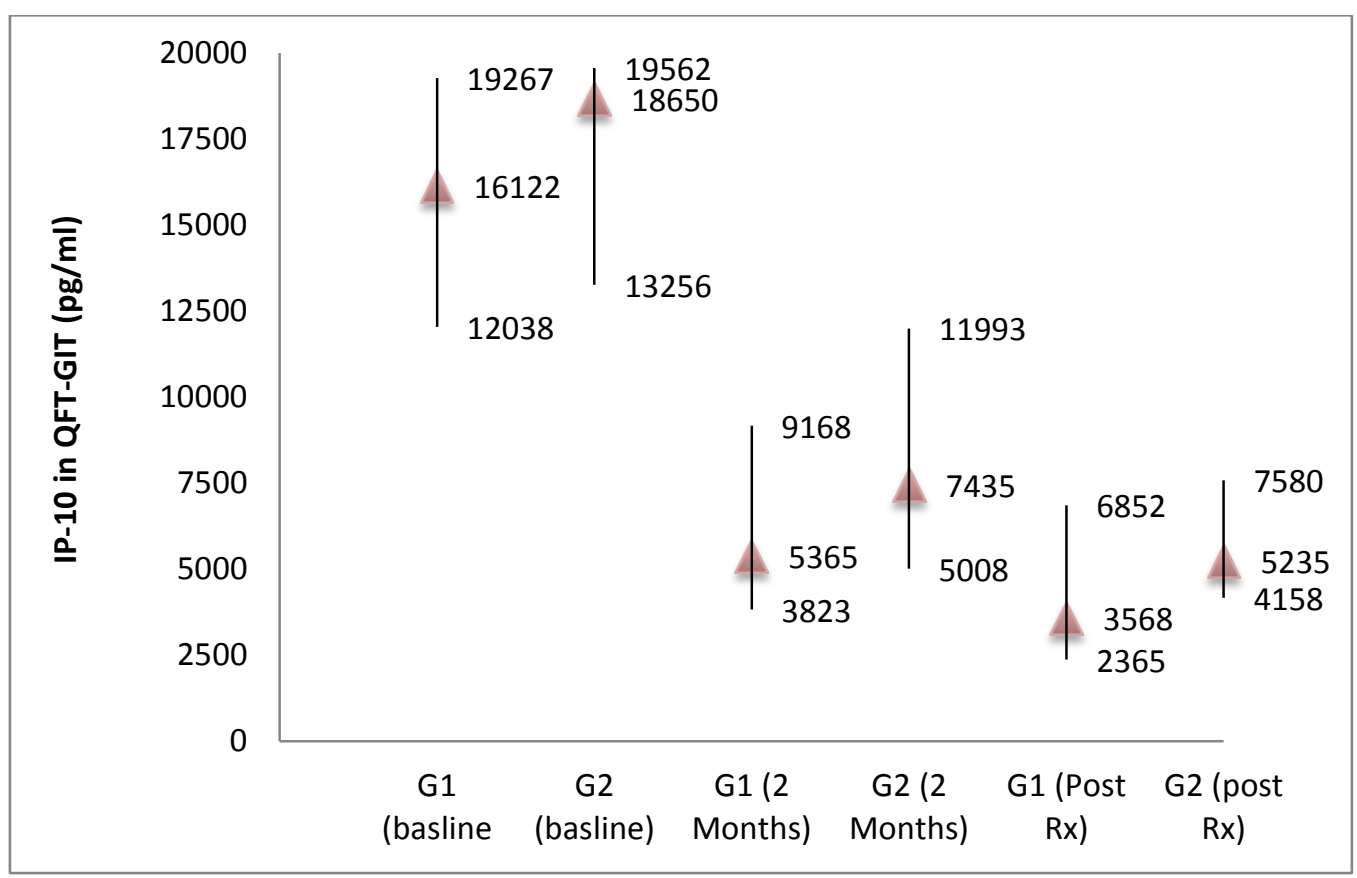

Figure (3): Boxplots showing medians and interquartile ranges of IP-10 levels in response to QFTGIT antigens in both patients groups at baseline sampling, at month 2 and at completion of therapy, ( $\mathrm{G}=\mathrm{Group}$ ).

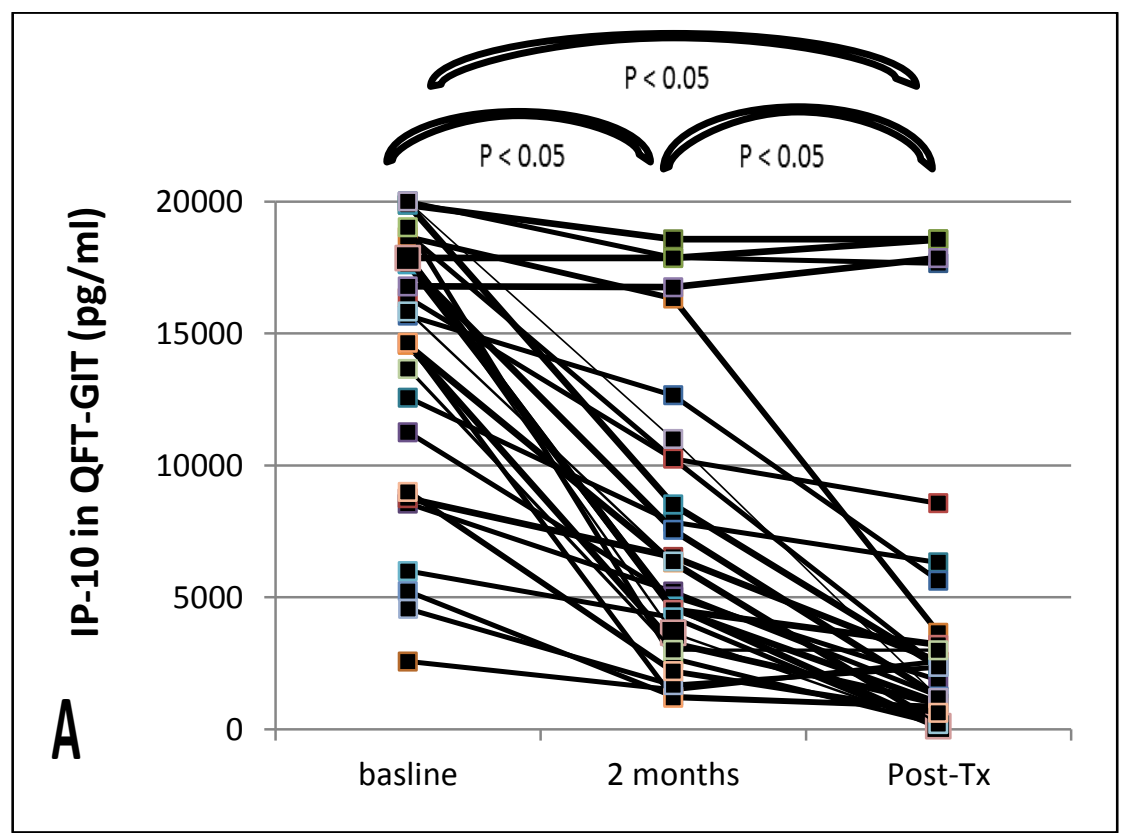




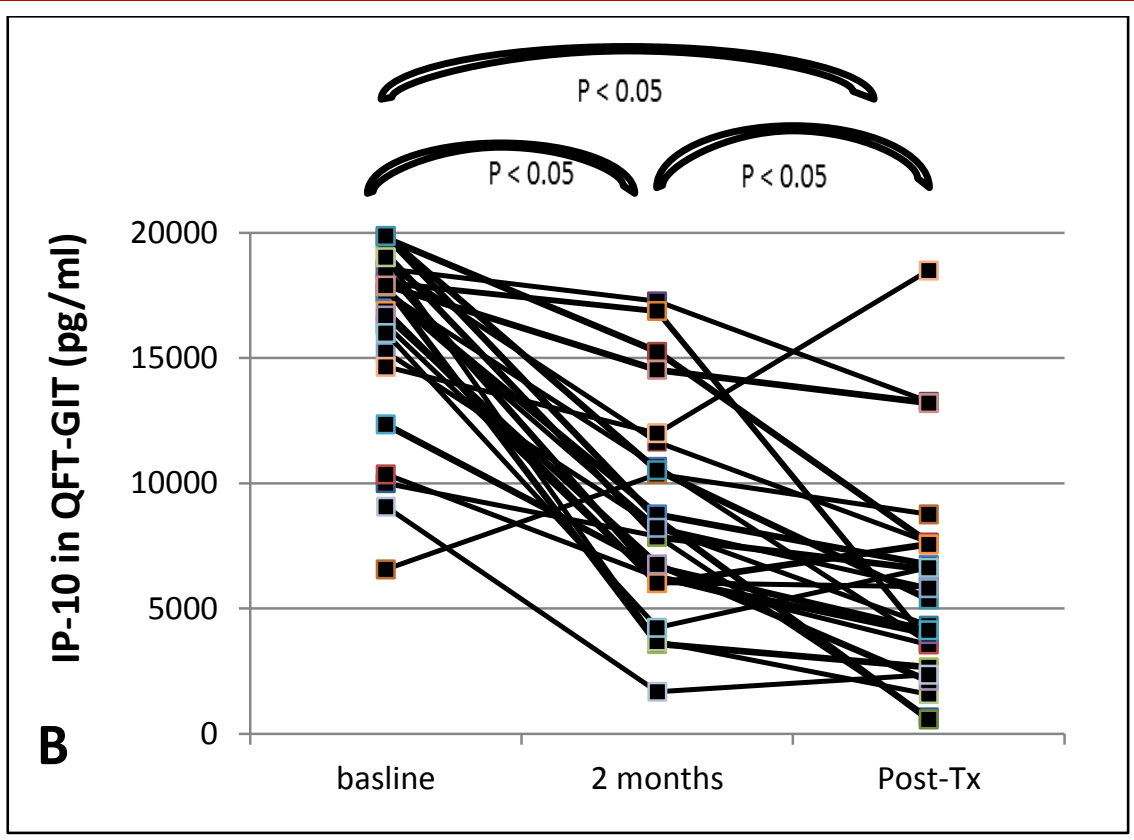

Figure (4): Longitudinal analysis of IP-10 levels in response to QFT-GIT antigens in both patients groups (group 1; A, group 2; B) at baseline sampling, at month 2 and at completion of therapy.

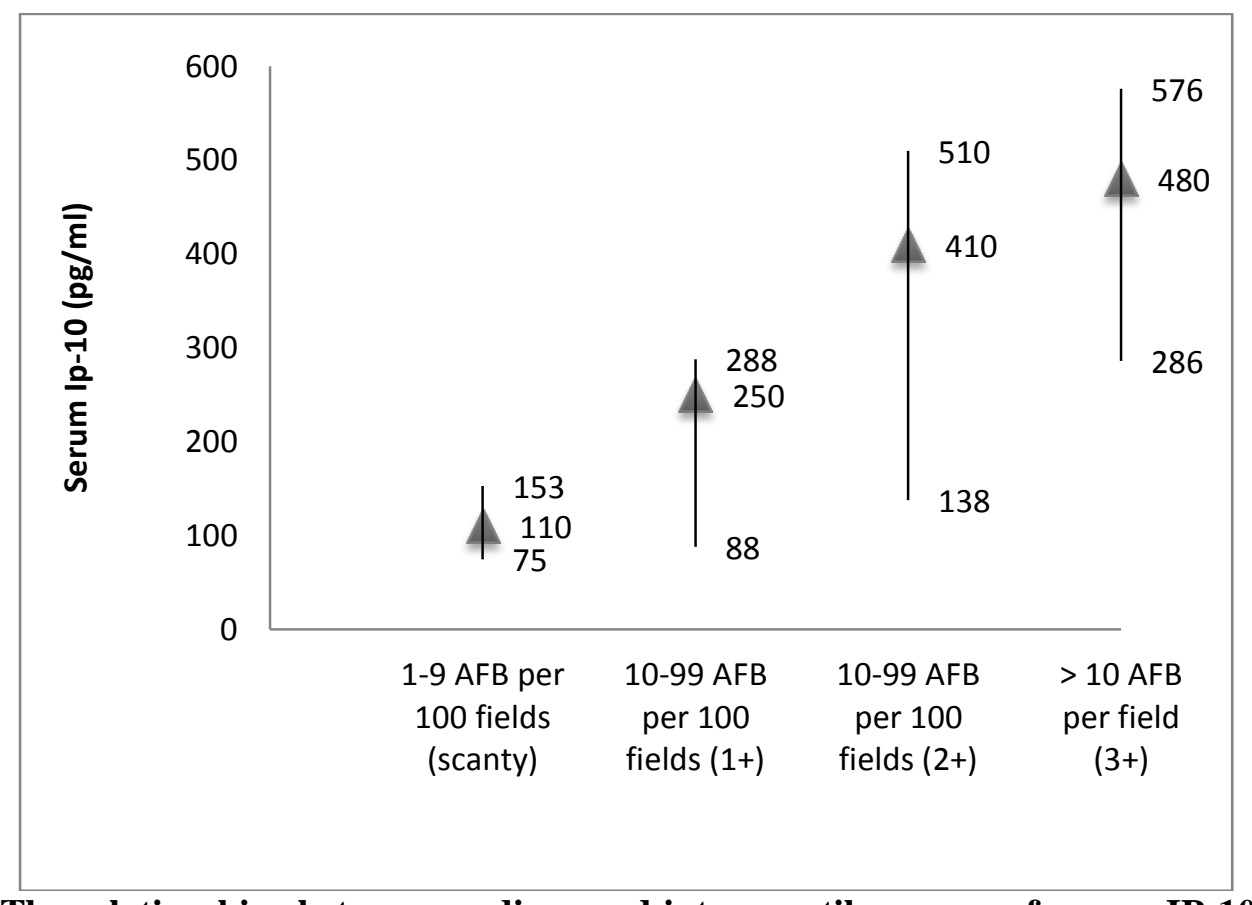

Figure (5): The relationships between medians and interquartile ranges of serum IP-10 and sputum smear grading for all studied patients at time of diagnosis.

\section{RESULTS}

This study included 55 patients with active pulmonary tuberculosis, which were positive by sputum smear and culture for AFB. They were 34 females and 21 males with mean age 38.2 \pm 2.6 years. They were categorized, according to their risk of disease relapse, into two groups 1 included 30 patients who had a low risk with mean age $39 \pm 14$, and group 2 included 25 patients with moderate to high risk for relapse 
with mean age was $37 \pm 10$. All patients were treated with first-line anti-TB drugs for at least 6 months and had negative smear and culture results at the completion of therapy. The baseline clinical and laboratory tests of both groups were shown in table 2 and all showed no statistical significant differences. Table 3 shows the treatment response based on microbiological and CXR findings, the duration of individual medication and the proportion of extended treatment course. The proportions of patients who need extended treatment course and those with sputum conversion or delayed resolution on CXR aftert month 2 of starting anti-TB treatment were significantly higher in the moderate/high-risk group than in the low-risk group $(\mathrm{P}<0.05)$.

Figures 1 and 2 shows the longitudinal analysis of serum IP-10 of patients in both patient groups before the start of antituberculous drugs, at month 2 and until completion of therapy. Median levels of serum IP-10 were not significantly different between both groups at baseline, at month 2 or after treatment. However, in the lowrisk group, the pre-tretment medians of serum IP10 levels decreased significantly with time from $203 \mathrm{pg} / \mathrm{ml}$ to $102 \mathrm{pg} / \mathrm{ml}$ at 2 months and to 71 $\mathrm{pg} / \mathrm{ml}$ after the completion of anti-TB treatment (P $<0.05)$. In comparison, the change in serum IP10 levels at baseline, 2 months and treatment completion in the moderate/high-risk group was also statistically significant (medians were 223 $\mathrm{pg} / \mathrm{ml}$ at baseline, $118 \mathrm{pg} / \mathrm{ml}$ at 2 months, and 78 $\mathrm{pg} / \mathrm{ml}$ Post-Tx, $\mathrm{P}>0.05$ ).

Figures 3 and 4 shows the longitudinal analysis of IP-10 levels in response to QFT-GIT antigens of patients in both patient groups before the start, at 2 months and at the end of TB therapy. Medians of IP-10 in response to QFT-GIT antigens were not significantly different between the low-risk and moderate/high-risk before starting the therapy, however in low-risk group, medians of IP-10 in response to QFT-GIT antigens at baseline decreased significantly from $16122 \mathrm{pg} / \mathrm{ml}$ to $5365 \mathrm{pg} / \mathrm{ml}$ at month 2 and to $3568 \mathrm{pg} / \mathrm{ml}$ after the end of anti-TB treatment $(\mathrm{P}<$ $0.05)$. On the other hand, medians of IP-10 in response to QFT-GIT antigen at baseline, 2 months and at treatment completion in the moderate/high- risk group were also statistically significant (medians were $18650 \mathrm{pg} / \mathrm{ml}$ at baseline, $7435 \mathrm{pg} / \mathrm{ml}$ at 2 months, and $5235 \mathrm{pg} /$ $\mathrm{ml}$ Post-Tx, $\mathrm{P}>0.05$ ).

Figures 5 shows that the relationships between median levels of IP-10 in serum at time of diagnosis were significantly higher in patients with high sputum smear grading than those with low sputum smear grading $(\mathrm{P}<0.05)$.

\section{DISCUSSION}

Tuberculosis remains one of the major causes of morbidity and mortality worldwide particularly in developing countries, however the understanding of its immunopathogenesis is still incomplete [13]. In patients receiving standard antituberculous regimen who have not documented resistance to first line antituberculosis drugs, the estimated relapse rate was $7 \%$ or less [14]. World Health Organization (WHO) still recommends sputum examination for AFB at month 2 after initiation of therapy for the possibility of the intensive phase extension. However, a recent study found that both sputum smear examination and mycobacterial culture have low sensitivity and modest specificity for predicting treatment relapse [15].

The monitoring role of QFT-GIT antigens for prediction of anti-TB treatment response has not extensively been investigated. Various studies that assessed the secretion of IFN- $\gamma$ in response to QFT-IT reported conflicting results among patients treated for active pulmonary TB. Some studies $[16,17,18]$ showed a significant decrease of IFN- $\gamma$ levels at end of therapy compared to the baseline, while other studies [19,20] revealed insignificant variation in IFN- $\gamma$ secretion after effective therapy. This extreme controversy may warrant the value of QFT-GIT antigens in the TB treatment monitoring process. Recently, some reports [21,22] proposed that sequential measurements of IFN- $\gamma$-inducible protein-10 in response to QFT-GIT antigens or in the blood can be a more useful in the monitoring of TB activity and of the expected results of therapy.

In this study, we investigated the efficacy of IP-10 in patients with active pulmonary TB as a sign of response to TB standard therapy and whether IP-10 as a biomarker could be affacted with the risk of cavitation and/or a positive sputum smear after 2 of starting anti-TB medications. We found that serum IP-10 and IP-10 secretion in response to QFT-GIT antigens may be useful tools to monitor the 
response of the standard 6-month regimen. We demonstrated that the decreasing levels of IP-10 were not depended on the risk of relapse. The patients in both groups had significant drop in IP-10 levels in serum and QFT- GIT tubes after starting anti-TB treatment and throughout the 6-month treatment course.

In compatible with our study, Azzurri et al.[23] who reported that serum IP-10 was correlated well with the treatment efficacy in patients with pulmonary TB. In addition, Chen et al.[8] suggested that serial determinations of serum IP-10 may be used to identify patients with rheumatoid arthritis who are developing active TB during TNF- $\alpha$ treatment. Riou et al. [21] also reported similar results. They found that the plasma concentrations of IP-10 were significantly reduced upon TB treatment, regardless of HIV status. Su et al. [24] reported a significant difference in plasma IP10 between early and late treatment responders based on chest radiographs after 2 months of intensive anti-TB treatment. They suggest that the relatively small decline in IP-10 levels in their moderate-/highrisk group may be related to the sustained inflammation and cytokine activation in this group. Another study, Goletti et al. [25] showed that the IFN- $\gamma$ secretion but not the IP-10 response to Mycobacterium tuberculosis region of difference (RD1) selected peptides is associated with active TB with a higher specificity than QFT-IT. The study had some limitations; subjects were selected according to a certain TB status; beside the design was a case control study that is not ideal for diagnostic evaluations.

\section{CONCLUSION}

Interferon-inducible Protein-10 (IP-10) in serum or in response to QFT-GIT antigens was a useful biomarker for monitoring the efficacy of the standard regimen in patients with active pulmonary TB.

\section{REFERENCES}

1. World Health Organization (2014): Global Tuberculosis Report 2014, (WHO/HTM/TB/2014.08). Geneva, Switzerland.

2. Chaisson R, Churchyard G (2010): Recurrent tuberculosis: relapse, reinfection, and HIV. J Infect Dis. Mar; 201(5):653-5. doi: 10.1086/650531.

3. Singla R, Srinath D, and Gupta S, et al., (2009): Risk factors for new pulmonary tuberculosis patients failing treatment under the Revised National Tuberculosis
Control Programme, India, Int J Tuberc Lung Dis 13, 521-526.

4. Blumberg H.M., Burman W.J., and Chaisson R.E., et al., (2003): American Thoracic Society/ Centers for Disease Control and Prevention/Infectious Diseases Society of America: treatment of tuberculosis, Am J Respir Crit Care Med 167, 603-662.

5. Johnson J, Hadad D, and Dietze R, et al. (2009): Shortening treatment in adults with noncavitary tuberculosis and 2-month culture conversion. Am J Respir Crit Care Med; 180(6):558-63.

6. Kabeer BS, Sikhamani R, and Raja A. (2010): Comparison of interferon gamma and interferon gamma-inducible protein-10 secretion in HIVtuberculosis patients. AIDS; 24:323-5.

7. Kabeer BS, Raja A, and Raman B, et al. (2011): IP-10 response to RD1 antigens might be a useful biomarker for monitoring tuberculosis therapy. BMC InfectDis; 11:135.

8. Chen YC, Chin $\mathrm{CH}$, and Liu SF, et al. (2011): Prognostic values of serum IP-10 and IL-17 in patients with pulmonary tuberculosis. Dis Markers; 31(2):10110.

9. Kent PT, Kubica GP (1985): Public health microbiology, a guide for the level III laboratory. Centers for Disease Control, Division of Laboratory Training and Consultation, Atlanta, GA.

10. American Thoracic Society/Centers for Disease Control and Prevention, (2000): Diagnostic standards and classification of tuberculosis in adults and children. Am J Respir Crit Care Med; 161:1376-95.

11. Ralph AP, Ardian M, and Wiguma A, et al. (2010): A simple, valid, numerical score for grading chest $\mathrm{x}$-ray severity in adult smear-positive pulmonary tuberculosis. Thorax; 65:863e9.

12. World Health Organization (2009): Guidelines for treatment of tuberculosis; fourth edition, WHO/HTM/TB/2009.420, Geneva; Switzerland.

13. O'Garra A, Redford P, McNab F, et al. (2013): The immune response in tuberculosis. Annu Rev Immunol.; 31:475-527.

14. Dye C, Watt C, Bleed D, et al. (2005): Evolution of tuberculosis control and prospects for reducing tuberculosis incidence, prevalence, and deaths globally. JAMA; 293:2767-75.

15. Horne D, Royce S, Gooze L, et al. (2010): Sputum monitoring during tuberculosis treatment for predicting outcome: systematic review and meta-analysis. Lancet Infect Dis. Jun; 10(6):387-94.

16. Carrara S, Vincenti D, Petrosillo N, et al. (2014): Use of a $\mathrm{T}$ cell-based assay for monitoring efficacy of antituberculosis therapy. Clin Infect Dis, 38(5):754756.

17. Katiyar SK, Sampath A, Bihari S, et al. (2008): Use of the QuantiFERON-TB Gold In-Tube test to monitor treatment efficacy in active pulmonary tuberculosis. Int J Tuberc Lung Dis, 12(10):1146-1152. 
18. Sauzullo I, Mengoni F, Lichtner M, et al. (2009): In vivo and in vitro effects of antituberculosis treatment on mycobacterial interferon- $\gamma \mathrm{T}$ cell response. PLoS One, 4(4):e5187.

19. Pai M, Joshi R, Bandyopadhyay $M$, et al (2007): Sensitivity of a whole-blood interferon- $\gamma$ assay among patients with pulmonary tuberculosis and variations in T-cell responses during anti-tuberculosis treatment. Infection, 35(2):98-103

20. Kobashi Y, Mouri K, Yagi S, et al. (2009): Transitional changes in T-cell responses to Mycobacterium tuberculosis-specific antigens during treatment. J Infect, 58(3):197-204.

21. Riou C, Perez Peixoto B, Roberts L, et al. (2012): Effect of Standard Tuberculosis Treatment on Plasma Cytokine Levels in Patients with Active Pulmonary Tuberculosis. PLoS ONE 7(5): e36886.

22. Hong J, Lee H, Kim S, et al. (2014): Efficacy of IP-10 as a biomarker for monitoring tuberculosis treatment. $\mathbf{J}$ Infect. Mar; 68(3):252-8.
23. Azzurri A., Sow O.Y., Amedei A., et al. (2005): IFNgamma-inducible protein 10 and pentraxin 3 plasma levels are tools for monitoring inflammation and disease activity in mycobacterium tuberculosis infection. Microbes Infect; 7:1-8.

24. Su WL, Perng WC, Huang $\mathrm{CH}$, et al. (2010): Association of reduced tumor necrosis factor alpha, gamma interferon, and interleukin-1beta (IL1beta) but increased IL-10 expression with improved chest radiography in patients with pulmonary tuberculosis. Clin Vaccine Immunol; 17(2):223e31.

25. Goletti D, Vincenti D, Carrara S, et al. (2005): Selected RD1 peptides for active tuberculosis diagnosis: comparison of a $\gamma$-interferon whole-blood enzymelinked immunosorbent assay and an enzyme-linked immunospot assay. Clin Diagn Lab Immunol, 12(11):1311-1316. 